\title{
Renal Corpuscle
}

National Cancer Institute

\section{Source}

National Cancer Institute. Renal Corpuscle. NCI Thesaurus. Code C33456.

A component of the nephron comprising two structures: Bowman capsule and the glomerulus, and which is responsible for the filtration of plasma. 